\title{
Effect of Polymerization of Aniline on Thermal Stability, Electrical Conductivity and Band Gap of Graphene Oxide/PolyanilineNanocomposites
}

\author{
Asim Riaz ${ }^{1}$, Adil Usman ${ }^{1}$, Muhammad Faheem ${ }^{2}$, Zakir Hussain ${ }^{1, *}$, Ahmad Nawaz Khan ${ }^{1}$, \\ Shahid Soomro ${ }^{3}$ \\ ${ }^{1}$ School of Chemical and Materials Engineering (SCME), National University of Sciences \& \\ Technology (NUST), Sector H-12, 44000 Islamabad, Pakistan \\ ${ }^{2}$ University of Azad Jammu and Kashmir Muzaffarabad, Pakistan \\ ${ }^{3}$ Bremer Pharma GmBH, Werkstr. 42, 34414 Warburg, Germany \\ *E-mail: zakir.hussain@ @scme.nust.edu.pk
}

doi: $10.20964 / 2017.03 .20$

Received: 7 December 2016 / Accepted: 31 December 2016 / Published: 12 February 2017

Effect of the polymerization of aniline on graphene oxide (GO) surface on the thermal stability, electrical and optical properties of GO has been studied through its chemical functionalization with aniline and polyaniline. GO obtained through liquid $e x$-foliation of graphite flakes was further covalently coupled with aniline and PANI through imidation protocol in order to extend its piconjugation and to study its effect on opto-electronic properties. Band gap of pristine as well as chemically modified GO was calculated through UV-Vis spectroscopy. It was found that after functionalization, band gap of GO decreased from $3.82 \mathrm{eV}$ to $2.87 \mathrm{eV}$. Furthermore, the conductivity of GO increased from $96.04 \mathrm{~S} / \mathrm{cm}$ to $139.87 \mathrm{~S} / \mathrm{cm}$ after modifying with PANI. XRD, FTIR, TGA and elemental analysis data have supported the difference in the properties of modified GO with aniline and PANI. Finally, TGA analysis showed that the thermal stability of GO was enhanced up to $20 \%$ due to the addition of PANI.

Keywords: 2D materials, Chemical synthesis, Electrochemical measurements, Polymerization, exFoliation.

\section{$\underline{\text { FULL TEXT }}$}

(C) 2017 The Authors. Published by ESG (www.electrochemsci.org). This article is an open access article distributed under the terms and conditions of the Creative Commons Attribution license (http://creativecommons.org/licenses/by/4.0/). 